\title{
Revisitando a Crítica de Bruner: O Desvio da Proposta Cognitivista nos Estudos de Solução de Problemas
}

\author{
Maria Isabel da Silva Leme ${ }^{1}$ \\ Universidade de São Paulo
}

\begin{abstract}
Resumo
A crítica feita por Bruner que a Psicologia Cognitiva tornou-se tecnicista e fragmentada é analisada por meio de uma revisão das pesquisas sobre transferência de solução de problemas, focalizando-se nestas os objetivos, metodologia e resultados obtidos. A conclusão é a de que a crítica procede, na medida em que se observa nas pesquisas cognitivistas um maior rigor metodológico do que teórico, revelado na ausência de uma definição precisa de transferência, produzindo resultados fragmentados. Observase ainda, uma visão tecnicista do processo, investigado, geralmente, como desempenho de estudantes universitários, resultado de manipulação de estímulos e avaliado em uma única sessão experimental.

Palavras-chave: Psicologia Cognitiva; transferência de solução de problemas; raciocínio analógico.
\end{abstract}

\section{Bruner's Criticism Revisited: Cognitivist Diversion on Problem Solving Studies}

\begin{abstract}
Bruner's claim that cognitivism has become fractionated and technicalized was examined by a review of the research on problem solving transfer. Objectives, methodology and results were analyzed leading to the conclusion that the claim is justified. The cognitivist investigations examined were methodologically very controlled in their experimental conditions, but the same rigor was not observed in theoretical matters, such as a precise definition of transfer, producing dispersed data. Also, a strong emphasis in investigating transfer as a result of stimulus manipulations was found evaluated in single session investigations, and considered only as performance of undergraduates, revealing an empiricist view of the process.

Keywords: Cognitive psychology; problem solving transfer; reasoning by analogy.
\end{abstract}

A presente pesquisa tem por objetivo analisar em que medida procede a crítica feita por Bruner (1990/1997) de que a revolução cognitivista tornou-se tecnicista e fragmentada, tomando um rumo diverso daquele que inicialmente havia se proposto perseguir. Segundo Bruner, o objetivo inicial não era reformar o behaviorismo, mas sim substituí-lo, resgatando a questão do significado como um conceito central na investigação psicológica: "não estímulos e respostas, não o comportamento claramente observável, não os impulsos biológicos e sua transformação, mas o significado." (p.16). Escolhemos a transferência de solução de problemas para a análise desta crítica, por duas razões: a investigação deste tema é uma preocupação antiga na Psicologia, pois, já na escola da Gestalt, enfatizava-se o estudo do processo de solução de problemas, dada sua importância para lidar com situações novas (Wertheimer, 1959). Nesta mesma perspectiva, a Psicologia Cognitiva, desde seu início, privilegiou este tema, procurando desvendar os processos que tornam possível ao ser humano adaptar conhecimentos adquiridos a uma outra situação. A segunda

\footnotetext{
${ }^{1}$ Endereço para correspondência: Instituto de Psicologia, USP, Av. Prof. Mello Moraes, 1721, 005508-900, São Paulo, São Paulo. Fone: (11) 38184355 , Fax: (11) 8138895.E-mail:milm@usp.br
}

razão vem da constatação de uma ênfase crescente no desenvolvimento da capacidade de solução de problemas nos alunos contida nas propostas educacionais atuais. Os Parâmetros Curriculares Nacionais (PCNs, 1998) justificam o desenvolvimento desta capacidade entre os objetivos do ensino fundamental : "Ao aprender a resolver problemas e construir atitudes em relação às metas que quer atingir nas mais diversas situações de vida, o aluno faz aquisições dos domínios cognitivo e lingüístico que incluem formas de comunicação e de representação espaciais, temporais e gráficas" (p.73). Um exame das críticas aos sistemas educacionais de outros países como Japão e Coréia revela que, também nestes países, é preocupação o melhor desenvolvimento desta capacidade para a adaptação do aluno a mudanças sociais cada vez mais freqüentes (Fleury \& Mattos, 1992). Neste sentido, vamos examinar por meio da análise deste tema, em que medida a Psicologia tem condição de responder satisfatoriamente às necessidades da Educação, expressas nas propostas educacionais atuais.

\section{Definição do Objeto de Estudo}

Uma definição bastante aceita entre os pesquisadores da área sobre o que constitui um problema é a constatação 
da existência de uma situação, contendo um objetivo a ser alcançado, cujos meios para a sua consecução não estão inteiramente claros (Sternberg, 2000). A discussão sobre a pertinência desta definição a todo e qualquer tipo de problema foge ao propósito do presente trabalho, que é o de examinar os resultados que a pesquisa em Psicologia tem obtido no esclarecimento da questão, notadamente sobre transferência dos conhecimentos adquiridos na situação, em que tal definição mostra-se adequada.

Para tanto, pesquisamos a literatura da área nos últimos vinte e cinco anos, quando surgiram os primeiros trabalhos sobre transferência de solução de problemas. Nossa análise vai caracterizar os tipos de pesquisa realizados em termos da visão do fenômeno, ou seja, o referencial teórico escolhido para a colocação do objetivo, a conseqüente metodologia empregada e os resultados obtidos, enfatizando-se as pesquisas cognitivistas, objeto principal deste trabalho. Nesta perspectiva, apresentaremos uma caracterização mais detida do referencial cognitivista, em relação aos outros identificados, após uma breve exposição da metodologia que adotamos para a coleta dos dados. Passaremos, então, a descrever como os dados foram analisados, os resultados e conclusões a que chegamos.

\section{Metodologia para Coleta de Dados da Presente Pesquisa}

O critério seguido para a coleta da literatura disponível foi o de o objeto das pesquisas publicadas consistir em transferência de solução de problemas, independente do referencial teórico adotado. Para tanto realizamos vários tipos de busca nas fontes disponíveis, como por autores já conhecidos na área, título, palavra chave, citações de trabalhos. Obtivemos assim, uma amostra de 61 relatos de pesquisas, abrangendo o período de 1974 a 1999. Feita a leitura do material, procedeu-se ao levantamento dos aspectos de interesse para a presente pesquisa e sua respectiva categorização. Os seguintes aspectos serão objeto de análise: referencial teórico, objetivos, metodologia (abrangendo os sub-tópicos participantes, material e procedimento) e resultados obtidos. Iniciaremos pelos referenciais teóricos identificados, cognitivismo, behaviorismo e funcionalista, caracterizando como cada um define a transferência de solução de problemas.

\section{Referenciais Teóricos Identificados}

Acima do que seria de se esperar, de acordo com o levantamento realizado por Robbins, Gosling e Craik (1999), que analisaram as principais tendências teóricas das pesquisas nos últimos trinta anos, a quase totalidade
(91\%) das pesquisas levantadas no presente trabalho adota o paradigma cognitivista do processamento de informação sobre solução de problemas. Duas pesquisas adotam o referencial behaviorista e uma o funcionalista. Surpreendentemente, uma não faz qualquer menção ao referencial teórico, propondo-se esclarecer empiricamente aspectos envolvidos na solução por insight versus na por ensaio e erro. A presente análise irá focalizar mais detidamente as pesquisas realizadas na perspectiva cognitivista, em função do objetivo proposto na introdução. Entretanto, analisará, ainda que brevemente, as realizadas em outros referenciais, pelo interesse que representam seus resultados para um melhor conhecimento na área.

\section{Caracterização dos Principais Referenciais Teóricos Referencial Cognitivista}

O estudo da solução de problemas no referencial cognitivista foi iniciado por Newell e Simon (1972) com o programa de computador The General Problem Solver, elaborado para provar teoremas da Lógica. A concepção, tanto de solução de problemas, como de processamento cognitivo geral, era de funcionamento lógico, no sentido de ser orientado primordialmente por princípios de resolução gerais, regras, algoritmos e procedimentos, ordenados e seqüenciais. Em outras palavras, supunha-se que, de posse do princípio de resolução adequado e dos algoritmos e procedimentos necessários para sua implementação, o participante teria condição de resolver qualquer problema apresentado. Os fatores aprendizagem ou experiência anterior, assim como os conhecimentos resultantes das mesmas, eram pouco considerados. Entretanto, as pesquisas posteriores mostraram que participantes mais experientes têm um desempenho superior e mais rápido, e, também, que o participante iniciante vai melhorando seu desempenho com a repetição da tarefa (Mattos, 1992). O papel do conhecimento anterior no funcionamento cognitivo foi ficando cada vez mais evidente. No início da década de oitenta começaram a surgir pesquisas sobre transferência de solução de problemas, preservando a concepção de funcionamento cognitivo como processamento de informação, cujas etapas podem ser identificadas e estudadas, mas considerando que este processo pode também ser indutivo, guiado pela experiência específica com um tipo de problema. Os processos neste tipo de raciocínio, podem ser identificados, como nos outros, por serem relativamente invariantes na interação com o meio. Seriam especificados genericamente nas seguintes fases: representação - codificação da situação, processamento e comportamento. $O$ processo de transferência 
segue as seguintes fases: aquisição, quando o participante aprende a lidar com a situação problema, e transferência, quando aplica conhecimento adquirido na aquisição à outra situação, vista como similar em algum aspecto. Esta similaridade pode ser de dois tipos: superficial, os problemas partilham elementos em comum como enunciado semelhante, objetos a serem operados etc, ou estrutural, quando os problemas demandam o mesmo processo de resolução, mas os enunciados abordam conteúdos diferentes.

A transferência baseada em percepção de similaridade é denominada analógica e segue as seguintes etapas: representação do problema a ser resolvido, recuperação de um problema análogo na memória, mapeamento (comparação dos dois problemas), inferência do procedimento de solução e aplicação. Após o contato repetido com esta situação, é adquirido algum tipo de conhecimento genérico sobre o tipo de problema, denominado esquema. Ё justamente neste último aspecto que encontramos diferenças importantes na área. Para alguns pesquisadores, como Holyoak e Koh (1987), o processo de resolução de problemas análogos resulta na indução de um esquema daquele tipo de problema, contendo informações, tanto sobre o processo de resolução em termos de objetivos, como sobre características superficiais, como o contexto, enunciado etc. Já para outros, como Gentner (Holyoak \& Gentner, 1997), o que é abstraído da situação é o processo de resolução, o que é comumente chamado analogia estrutural, os problemas, fonte e o alvo, demandam as mesmas operações para serem resolvidos.

Como seria de se esperar, encontra-se assim, uma grande diversidade de concepções acerca do que é considerado como transferência, transferência analógica e os critérios que são adotados para definir dois problemas como análogos. Para alguns autores, a transferência ocorre quando um conhecimento adquirido é aplicado em uma outra área de conhecimento ou domínio, como por exemplo, o conceito de inibição em química e em biologia (Schunn \& Dunbar, 1996), enquanto, para outros, a transferência seria aplicação de conhecimento em uma situação nova no mesmo domínio, como, por exemplo, uma nova equação (Rickard, Healy \& Bourne Jr., 1994), ou uma nova série de dígitos alfanuméricos (Burns, 1996). Encontramos variações também sobre o que é considerado domínio diferente. Segundo a definição de alguns pesquisadores, o domínio muda quando se exige a aplicação de um tipo diferente de raciocínio, como o indutivo e o dedutivo (Phye \& Sanders, 1994), e não somente de conceitos, estratégias ou habilidades que se aplicam a diferentes áreas de

Psicologia: Reflexão e Crítica, 2001, 14(3), pp. 521-536 conhecimento, como vimos acima (Antonietti, 1991; Gick \& Holyoak, 1980). É possível que tais diferenças possam ser atribuídas ao surgimento da própria Psicologia Cognitiva, que não tem uma "paternidade" definida, como a Psicanálise ou o Construtivismo Piagetiano, que defina os constructos teóricos em investigação. Como pode ser depreendido das palavras de Bruner (1990/ 1997), a Psicologia Cognitiva surgiu a partir de uma insatisfação crescente de pesquisadores de diferentes temas, como memória, solução de problemas, formação de conceitos etc. com o paradigma behaviorista. Esta insatisfação centrava-se na investigação exclusiva de fenômenos psicológicos observáveis, resultando em dados de poder explicativo muito limitado. Neste sentido, propostas alternativas de investigação foram surgindo, no início, de modo esparso e enfrentando muita oposição, mas ganhando força à medida em que resultados foram sendo obtidos.

Em resumo, consideramos que uma pesquisa sobre transferência de solução de problemas foi realizada segundo o referencial cognitivista se tem como pressuposto que este é um processo deliberado, dirigido por um objetivo, que envolve etapas de processamento identificáveis e passíveis de investigação.

\section{Referencial Behaviorista}

Verificamos a existência de diversas tendências, diferentes quanto à maior aceitação da intervenção de processos subjetivos, no que se considera referencial behaviorista. Esta diferença também é encontrada em relação à solução de problemas, encontrando-se duas visões que se destacam: a de Skinner e a de seguidores de Hull.

A primeira adota a seguinte definição de solução de problemas: é uma questão para a qual não se tem resposta no momento (Skinner, 1966). Esta resposta, que não se diferencia de qualquer outra, é aprendida de acordo com os mesmos mecanismos que governam outras aprendizagens, ou seja, pelas suas contingências. São, então, aspectos importantes na solução de problemas: 1) as contingências ambientais do comportamento; 2) a construção de um modelo do ambiente reforçador, geralmente explicitada em regras ou até conselhos; 3) a indução, quando não há explicitação das contingências em regras, só exposição às mesmas, e, finalmente, 4) a dedução. Esta última, seria a construção do estímulo discriminativo, ou regras de segunda ordem, derivadas da descoberta do sucesso de outras práticas, ou do exame dos sistemas de manutenção de contingência descritos pelas regras de primeira ordem. Skinner (1989) esclarece a diferença entre comportamento governado por regra daquele que é modelado pelas contingências: o primeiro 
seria uma resposta a um tipo específico de estímulo verbal, sendo mais racional, abstrato e público. Já o segundo seria mais individual, privado, intuitivo e ligado ao plano concreto.

Na perspectiva hulliana, a solução de problemas pode ser concebida como qualquer aprendizagem que não seja de condicionamento reflexo, resultando na produção de hierarquias de famílias de hábitos ou respostas. Em uma perspectiva um pouco mais estreita, seus seguidores propõem que a resolução de um problema envolve um desempenho específico para a consecução de um objetivo, para o qual o indivíduo está motivado. Este dispõe de várias respostas associadas ao mesmo objetivo, disponível de acordo com um mecanismo antecipatório, ativado pelas instruções ou características percebidas no problema. O indivíduo coloca em prática a resposta mais ativada, e só mobiliza a próxima no caso de fracasso da primeira. A solução de problemas dá origem ao que é chamado de família de hábitos composta, na qual uma resposta que era eliciada por uma classe de estímulos passa a ser eliciada por outros das hierarquias próximas, pela ação da generalização mediatizada (Maltzman, 1965).

Em suma, consideramos que uma pesquisa sobre transferência de solução de problemas foi realizada segundo o referencial behaviorista se o processo é considerado, como qualquer outro, controlado por contingências ambientais. A transferência envolve generalização de estímulos, que passam a controlar o comportamento em uma situação diferente daquela em que foi adquirido.

\section{Referencial Funcionalista}

A análise das condutas em solução de problemas neste referencial dá continuidade aos trabalhos piagetianos, investigando funcionalmente como os conhecimentos gerais identificados por estes são colocados em prática. Nesta perspectiva, as atividades de solução de problemas não são consideradas independentes da teoria piagetiana sobre o sujeito epistêmico. Pelo contrário, é dentro deste quadro de referência geral, que as análises das condutas fazem sentido. Por outro lado, trata-se, sobretudo, de compreender como se constroem novos conhecimentos a partir do processo de solução, e como podem ser transferidos para outras situações. Enquanto Piaget tinha por objetivo descrever e explicar os componentes universais do desenvolvimento, a macrogênese do sujeito epistêmico, a abordagem funcionalista visa investigar a microgênese do sujeito psicológico, centrando-se sobre a dinâmica das condutas, procurando trazer elementos para responder as seguintes perguntas: Que significado o participante dá à tarefa? Quais são os meios escolhidos?
Quais são as representações importantes para a tarefa? Quais são os controles exercidos pelo participante? (Coulet, 1999). Em resumo, o referencial funcionalista investiga a transferência de solução de problemas como a aplicação prática de condutas e conhecimentos, em função do nível de desenvolvimento e dos mecanismos que provocam as mudanças cognitivas.

\section{Objetivos das Pesquisas Segundo seu Referencial Teórico}

Nas pesquisas realizadas segundo o referencial cognitivista foram identificados os seguintes objetivos: 1)testar a influência de manipulações experimentais como tipo de tarefa, tipo de treino, intervalo entre aquisição e teste de transferência etc nas etapas de aquisição ou transferência (72\%); 2 ) avaliar a influência do conhecimento anterior, como diferentes níveis de expertise, conhecimento factual $(14 \%) ; 3)$ esclarecer o funcionamento e a importância relativa dos processos já identificados como envolvidos em solução de problemas como, por exemplo, entendimento (representação), mapeamento, conhecimento procedural versus declarativo; descoberta da regra; acessibilidade do conhecimento, uso de diferentes estratégias, interpretação da questão, tipo de esquema formado na aquisição (10\%); e, 4) investigar a influência de processos metacognitivos (4\%). Dentre as pesquisas realizadas segundo o referencial behaviorista vamos encontrar uma que tem por objetivo verificar o papel da generalização sobre a transferência, e outra, que visa a investigar o papel de uma pista retardada sobre a transferência.

No referencial funcionalista encontramos uma pesquisa que tem por objetivo investigar como o conhecimento prévio é colocado em prática em uma tarefa, contendo informações para a sua resolução. Finalmente, a pesquisa sem referencial tem por objetivo investigar diferentes tipos de aquisição sobre a transferência, visando a esclarecer, ainda, aspectos envolvidos na solução por insight e por ensaio e erro.

Concluindo: observa-se nas pesquisas cognitivistas uma forte ênfase na busca de definição de condições de aquisição e teste que favorecem a transferência, facilitando o processo, em detrimento de outros, mais coerentes com a proposta inicial, como o conhecimento anterior do participante sobre o domínio do problema, ou sobre a própria cognição. Conforme ficará melhor evidenciado nas próximas seções, tal ênfase em um tipo de questão de pesquisa tem como conseqüência investigações altamente controladas experimentalmente, cuja validade ecológica mereceria maior reflexão. Já as pesquisas realizadas segundo outros referenciais são consistentes 
com as suas propostas: no behaviorismo as condições ambientais são de extrema relevância, assim como o conhecimento resultante do desenvolvimento cognitivo e das interações do participante com a tarefa é para o referencial funcionalista.

\section{Método de Pesquisa segundo o Referencial Adotado Participantes}

Nas pesquisas realizadas segundo o referencial cognitivista, observamos que a grande maioria $(86 \%)$ teve como participantes estudantes universitários, que estavam, geralmente, inscritos em curso de Psicologia Introdutório e que receberam créditos ou pagamento pela sua participação. Dentre as restantes, oito por cento tiveram como participantes alunos do ensino fundamental, valendo ressaltar que três destas investigações foram realizadas pelo mesmo grupo de pesquisa. Em quatro por cento das pesquisas foram participantes alunos do ensino médio, também duas realizadas pelo mesmo pesquisador. Encontramos ainda, pesquisas cujos participantes eram de duas faixas etárias diferentes: um por cento das pesquisas teve como participantes alunos universitários e alunos do ensino médio; e um por cento dos alunos do Ensino Fundamental e alunos do Ensino Médio. Em uma pesquisa de referencial behaviorista foram participantes alunos universitários e na outra, um adulto portador de deficiência mental. $\mathrm{Na}$ de referencial funcionalista foram participantes crianças e na sem referencial adultos. É digna de nota a presença tão predominante de participantes de uma só faixa etária nas pesquisas cognitivistas, principalmente quando mais da metade das investigações que estudaram participantes de outras idades foram realizadas pelos mesmos pesquisadores.

\section{Material}

Foram identificados dois tipos de problemas, usados aproximadamente na mesma proporção, nas pesquisas cognitivistas:

1) que demanda pouco conhecimento específico anterior como: os clássicos problemas da radiação de Duncker e seus análogos como o problema do general (15\%), Torre de Hanói e seus análogos como missionários e canibais $(12 \%)$, analogias verbais $(5 \%)$, abstração da regra regendo seqüências de letras ou números (5\%), a Tarefa de Wason (3\%), silogismos (3\%) interceptação de objeto em movimento ( $2 \%)$, julgamento sobre plausibilidade de uma explicação $(2 \%)$ ou decisões em situações fictícias ( $2 \%$ ), avaliação do uso de uma estratégia metacognitiva $(2 \%)$, uso de um princípio geral de equivalência $(2 \%)$. Total: $53 \%$.
2) que envolve conteúdo acadêmico como: aritmética ou matemática (31\%), outras áreas como física, computação, química, lógica etc (13\%). Total: 47\%.

Verifica-se assim, que mais da metade das pesquisas usa problemas considerados como demandando pouco conhecimento anterior. Entretanto, deve-se observar que uma proporção considerável, quase metade, usa problemas que demandam este tipo de conhecimento. Como veremos na próxima seção, nem sempre os procedimentos usados adequam-se a este tipo de problema.

Um aspecto importante a ser analisado é o tipo de similaridade existente entre os problemas usados na aquisição e teste. Observa-se, que em função do que já foi apontado acima, sobre as diferentes concepções acerca do que considerar como transferência, encontra-se, também, uma variedade de critérios para definir duas situações como análogas. Mais de um terço das pesquisas (37\%) usou problemas análogos estruturais, ou seja, problemas cujos enunciados diferiam nos elementos usados (domínio diferente de conhecimento etc), mas, cujo processo de resolução demandava as mesmas operações. Em 23\% das pesquisas foram usados os dois tipos de análogos, superficiais e estruturais, e em $9 \%$ das pesquisas, problemas definidos como isomorfos (similaridade superficial e estrutural). Em 16\% foi dada apenas alguma informação anterior, como afirmações sobre a situação abordada no problema do teste de transferência. Em 14\% não foram usados problemas análogos, por estarem medindo a influência de conhecimento anterior. Os materiais usados nas pesquisas behavioristas foram: estímulos visuais sem significado para a formação de classes de equivalência, e estímulos com significado para nomeação. O problema usado na pesquisa funcionalista foi uma adaptação da tarefa de seriação em uma simulação computacional de uma balança insensível. Na pesquisa que não faz menção ao referencial teórico, a tarefa consistia na solução de um jogo de inversão de triângulo, com um número limitado de movimentos.

\section{Procedimento segundo o Referencial Adotado}

Observa-se na maioria das pesquisas cognitivistas (75\%) o uso do mesmo tipo de procedimento para investigar a transferência: uma fase inicial de aquisição, não raro denominada treino, seguida de um teste para avaliar a transferência. Entretanto, observa-se boa variação no que é considerado aquisição: leitura do problema fonte já resolvido, resolução do fonte sem qualquer informação adicional até situações de instrução altamente estruturadas como aula, ou mesmo, cursos. Já 
na transferência encontra-se a tendência oposta. Mais da metade $(60 \%)$ só avaliou o desempenho dos participantes em um teste. Em um terço das restantes foi colhido relato verbal do processo de resolução, sendo que em $12 \%$ destas com apenas parte dos participantes, ou em algum estudo preliminar. Nas demais foram colhidas algumas outras medidas como resumos, questionários, ou mesmo, tipo de erro cometido. Os estudos em sua maioria (63\%) são conduzidos em grupo, sendo as instruções e as tarefas administradas em folheto. Entre os $27 \%$ que avaliaram os participantes individualmente, $41 \%$ dos mesmos foram realizados no computador. Poucas pesquisas (24\%) investigaram a transferência depois de transcorrido um intervalo de tempo da aquisição, sendo a maioria destas (75\%) em um intervalo de, no máximo, sete dias. Oito pesquisas realizaram somente um teste de transferência por medirem a influência de conhecimento anterior. Uma parte $(16 \%)$ das pesquisas que realizou aquisição e teste limitou o tempo de exposição na aquisição e de resolução no teste, $12 \%$ além de limitarem o tempo, deram instruções falsas acerca do objetivo do estudo e 18\% incluíram tarefas de outras pesquisas entre as experimentais, ou problemas não relacionados. Poucos autores informam o motivo desta inserção, geralmente, estudo de variáveis como variação de contexto entre aquisição e transferência. Em outras não é explicado o motivo da inserção de outras tarefas.

Outra variável muito pesquisada (45\%) é o efeito do fornecimento de uma pista sobre a utilidade do problema anterior ou do princípio de resolução. Encontramos variações também sobre: quantidade de análogos fornecidos; tarefas realizadas para facilitar a aquisição como aulas, resumos, respostas a questões e avaliação de características pessoais dos participantes como estilo cognitivo. Em resumo, pode-se identificar nas pesquisas cognitivistas o predomínio de um mesmo tipo de procedimento experimental, sendo aquisição e treino realizados geralmente na mesma sessão, avaliados pelo mesmo tipo de medida, desempenho, encontrando-se variação em aspectos como contexto, fornecimento de informação como dicas.

Quanto às pesquisas realizadas segundo outros referenciais, verificamos que as duas pesquisas caracterizadas como behavioristas foram conduzidas, individualmente, uma no computador e outra com o experimentador. $\mathrm{Na}$ realizada por universitários no computador, treino e teste foram feitos em quatro etapas em uma única sessão experimental. Foi considerado como indicativo de generalização e transferência, os participantes substituírem o feedback certo e errado por estímulos figurais neutros, treinados previamente como membros de uma classe de equivalência (Greenway, Doulgher \& Wulfert, 1996). No estudo realizado individualmente com o experimentador treino e transferência foram investigados em três experimentos, em que se variou o tipo de estímulo e o tempo de apresentação do feedback. A transferência foi definida como uma resposta antecipatória de um participante, portador de deficiência mental leve, em uma tarefa de pareamento de estímulos. A resposta antecipatória consistiria em pronunciar oralmente o nome do estímulo, antes da apresentação do estímulo discriminativo (Glat, Gould, Stoddard, \& Sidman, 1994).

A pesquisa classificada como funcionalista foi realizada individualmente em duas sessões no computador, após um período de familiarização com o mesmo. A transferência foi investigada entre duas simulações computacionais de tarefas estruturalmente isomórficas, assim definidas por requererem o uso de mesmas operações de comparação e ordenação, sendo, porém, a segunda mais complexa que a primeira, pela exigência de outras operações inferenciais (Shao \& Nguyen Xuan, 1994). A pesquisa que não faz referência a qualquer teoria testou os participantes individualmente, variando-se o processo de resolução, poder ou não manipular diretamente os objetos dos jogos e número de peças componentes a serem removidas para inverter $\mathrm{o}$ triângulo (Tsai, 1987).

Em conclusão, o que se observa nas pesquisas cognitivistas é uma forte presença de uma metodologia de investigação muito uniforme, em termos do tipo de participantes e procedimentos de avaliação de transferência muito similares entre si, resultando em maior ênfase em um só tipo de medida, cujas implicações serão discutidas na seção de conclusão do trabalho. Por outro lado, uma diversidade muito grande de situações em termos de exposição ao conhecimento a ser empregado no teste, no tipo de problema usado, não só quanto ao conhecimento demandado, mas das informações disponíveis, resultantes de uma ausência de consenso sobre o que deve ser considerado como indicativo de transferência. O número de pesquisas realizadas segundo outros referenciais é pequeno para estabelecer comparações. Entretanto, observa-se nas mesmas, consistência entre referencial adotado e metodologia empregada para investigar a hipótese em estudo.

\section{Resultados segundo o Referencial Adotado Referencial Cognitivista}

A análise dos resultados encontrados nas pesquisas examinadas revelou-se uma tarefa complexa, em vista da existência de fatores já apontados acima, que dificultam o agrupamento dos mesmos. O primeiro diz respeito ao aspecto já analisado anteriormente, os objetivos de mais 
da metade das pesquisas eram o de testar a influência de diferentes manipulações experimentais sobre o desempenho no teste de transferência, encontrando-se variações desde o tipo e número de análogos até o intervalo entre as diferentes etapas experimentais. Outra dificuldade encontrada é de ordem conceitual, relativa à diversidade de concepções sobre o que é considerado como transferência, como já apontamos acima. Esta diversidade tem como conseqüência resultados até contraditórios, como veremos a seguir. Tendo em vista estes aspectos, vamos descrever em primeiro lugar os resultados encontrados sobre transferência específica, os quais serão resumidos na Tabela 1. Iniciaremos, então, por uma análise acerca dos resultados encontrados sobre o papel da similaridade dos problemas, um dos fatores mais investigados, como seria de se esperar, que ilustra as dificuldades encontradas.

\section{Influência da Similaridade}

A principal conclusão a que chegam as pesquisas cognitivistas é que a ocorrência de transferência depende da percepção do participante que existe similaridade entre a situação de aquisição e a de teste, de modo a aplicar o raciocínio analógico, isto é, o estabelecimento de inferências acerca das relações percebidas entre os elementos presentes em duas situações. Inicialmente, alguns autores esperavam que o processo ocorresse automaticamente, por ser baseado no raciocínio indutivo (Gick \& Holyoak, 1980). No entanto, constatou-se que na situação de pesquisa, a percepção de relação entre os problemas definidos como análogos, mas de domínios diferentes, dificilmente ocorre de modo espontâneo. Quando ocorre, é, em geral, restrita a menos da metade dos participantes (Chen, 1995; Gick \& Holyoak, 1980).

Tendo em vista estes resultados, as pesquisas passaram a investigar se a similaridade superficial, enunciado de problemas contendo elementos e situações semelhantes têm algum efeito sobre a transferência. Os resultados evidenciaram que a similaridade superficial ajuda na recuperação do análogo, mas não garante a transferência porque se restringe à facilitação ao acesso e comparação entre os dois problemas (Hesse \& Klecha, 1990; Holyoak \& Koh, 1987; Ross, 1987). Mesmo neste nível, é necessário separar a influência dos diversos componentes sobre a recuperação, pois alguns resultados mostraram que a similaridade entre enunciados favorece o acesso ao análogo, e entre objetos a recuperação das operações (Ross, 1989). Em outras palavras, a similaridade faz com que o participante lembre de uma situação semelhante, retire informação relevante até das operações necessárias, estabeleça comparações, mas não garante que o

Psicologia: Reflexão e Crítica, 2001, 14(3), pp. 521-536 participante saiba aplicar o procedimento de resolução, fazer as adaptações necessárias nos mesmos (Holyoak \& Koh, 1987). Neste aspecto da adaptação e aplicação, encontramos alguns resultados contraditórios, talvez decorrentes da adoção de perspectivas diferentes sobre o fenômeno. Alguns resultados indicaram que os processos de adaptação e aplicação seriam garantidos pela similaridade total, superficial e estrutural, isto é, enunciado contendo situações e elementos semelhantes e operações idênticas (Bard e cols., 1995; Patrick \& Haines, 1988; Rickard e cols., 1994), levando alguns a caracterizar, com razão, o processo como generalização (Reed, Dempster \& Ettinger, 1985). Por outro lado, encontraram-se evidências de que não é necessária similaridade total para a transferência, que esta pode ocorrer mesmo quando a analogia é parcial, limitada à correspondência entre objetos dos problemas (Ross, 1989), ou estrutural (Gentner \& Holyoak, 1997). Em outras palavras, contendo as mesmas operações a serem realizadas, inclusive na mesma ordem (Antonietti, 1991). Porém, quando limitada ao princípio de resolução geral, é insuficiente para a ocorrência da transferência (Chen, 1995; Simon \& Hayes, 1976). O fornecimento de uma instrução sobre como resolver um problema só é eficaz quando explicita os operadores a serem usados (Kotovsky \& Simon, 1990). É interessante observar que não se encontra consenso até sobre a ineficácia do principio geral, considerado mais eficiente que o contexto similar na promoção de transferência (Hesse \& Klecha, 1990). O contexto similar facilita a recuperação, mas pode resultar em rigidez funcional, já identificada na Escola da Gestalt por Luchins (1942) e Duncker (1945). É importante observar ainda, que a similaridade parcial, restrita ao nível superficial, pode induzir um processo chamado de transferência negativa, o uso inadequado do processo de resolução de um problema em outro (Novick, 1988). O que se pode concluir é que a similaridade superficial ajuda na recuperação do análogo, mas são necessárias outras informações acerca do processo de resolução, cujo nível de generalidade não se encontra bem estabelecido. Tendo examinado o papel que os tipos de similaridade tem sobre a ocorrência completa, parcial ou até negativa de transferência, cabe examinar os resultados de pesquisa que podem explicar tais efeitos. São as pesquisas que investigaram desde o que é retido na memória do solucionador e avaliado na própria situação de pesquisa, até o conhecimento de longo prazo que o participante tem, e que interage com as características do problema em investigação. 


\section{Papel do Conhecimento: Informação Retida na Situação Experimental}

Como veremos a seguir, também não se encontra consenso sobre o que é retido na memória entre as etapas de aquisição e transferência. Alguns resultados apontam para a retenção de informação específica, diretamente relacionada ao problema, enquanto outros na direção de conhecimento mais genérico. Em problemas como a Torre de Hanói, por exemplo, verificou-se que a informação mais simples é retida na memória, que facilita ou dificulta a aplicação de operadores e estratégias, dependendo da ordem de apresentação (Reed, Ernst \& Banerjii, 1974). No caso de conceitos, foi verificada retenção de informação específica entre aquisição e teste: contexto de aquisição e similaridade (Stein, Way, Benningfield \& Hedgecough,1986). Alguns resultados encontrados por Anderson e Fichman (1994) sobre o tipo de informação retida, que auxiliava seus participantes na transferência, explicam a retenção de conhecimento mais específico e contextualizado. Segundo os autores, o que é retido e, portanto, transferível, é conhecimento declarativo (saber que). Este é transferível para procedural (saber como), mas não o inverso. No caso de aprendizagem de uma regra, por exemplo, o que se abstrai dela é apenas um conhecimento ou representação declarativa da mesma, e não a sua aplicação, como ocorre quando se aprende a partir de exemplos. A transferência ocorre gradualmente, de aspectos específicos para um conhecimento geral. Este processo pode ser acelerado, caso se enfatize abstração de relações na aquisição, levando a uma melhor elaboração do conhecimento declarativo, o que facilita a transferência (Pennington, Nicolitch \& Rahm, 1995).

Estes últimos resultados sugerem que alguns tipos de aquisição favorecem a retenção de conhecimento mais genérico, na própria situação experimental, explicando, talvez, as diferenças encontradas. Isto porque, para alguns autores, o que é retido na memória é um tipo de informação mais geral e organizada, à qual chamam esquema. A exposição a problemas similares faz com que seja formado um esquema acerca daquele tipo de problema, embora o mesmo possa ser formado também na transferência, facilitando as posteriores (Spellman \& Holyoak, 1996). Também não se encontra consenso sobre o tipo de conhecimento contido no esquema: para alguns pesquisadores o esquema inclui tanto conhecimento declarativo como procedural, enquanto que para outros só procedural (Gentner \& Holyoak, 1997). Várias pesquisas encontraram evidências de facilitação da transferência analógica pela abstração de esquema (Bernardo, 1984; Catrambone, 1994; Catrambone \&
Holyoak, 1989; Clement, 1994; Cooper \& Sweeler, 1987; Gick \& Holyoak, 1983) e, também, lógica, pelo uso de um esquema já existente (Kroger, Cheng \& Holyoak, 1993). A formação do esquema não só facilita a transferência, como precede a automatização de aplicação da regra (Cooper \& Sweeler, 1987). O fornecimento do princípio de resolução teria justamente o efeito de favorecer a abstração do esquema porque ajuda na eliminação da informação desnecessária, às vezes até prejudicial (Hesse \& Klecha, 1990). A qualidade do esquema tem maior influência sobre a transferência do que uma comparação bem feita (Novick \& Holyoak 1991).

\section{Fatores que Favorecem Formação de Esquema}

Alguns fatores que favorecem a formação do esquema, possivelmente por propiciarem maior processamento da informação, foram identificados: atividades realizadas durante a aquisição como memorizar, resumir, comparar as similaridades entre problemas análogos já resolvidos (Clement, 1994), estudo com pista (Bernardo, 1984), exposição a exemplos variados de problemas (Paas, 1992; Paas \& Van Merrinboer, 1994), assim como a exposição a mais de um análogo (Cooper $\&$ Sweeler, 1987), principalmente quando acompanhado do princípio de resolução, de um diagrama representativo da solução, ou de instruções para resumir similaridades (Gick \& Holyoak, 1983). A formação do esquema foi avaliada em termos da solução dada e, também, das informações contidas no resumo pedido (Gick \& Holyoak, 1983; Novick \& Holyoak, 1991). Esquemas superiores em crianças são obtidos por meio de comparações (mapeamento) que enfatizem os aspectos relacionais (Pierce, Duncan, Gholson \& Khamni, 1993). Porém, o sucesso neste processo depende, também, do nível de desenvolvimento cognitivo, pois crianças mais jovens, abaixo da terceira série, só abstraem o esquema quando o domínio do problema é familiar (Pierce, Crain, Gholson, Smither \& Rabinovitz, 1996). Caso contrário, parecem guiar-se mais por scripts das situações, do que por comparações entre as mesmas. Ao que tudo indica, tais comparações ocasionam sobrecarga na memória ao exigir codificação de operações, restrições, etc. (Pierce \& Gholson, 1994). Estes resultados mostram então que, a despeito dos dados acerca de retenção mais específica, encontrados pelas pesquisas anteriormente citadas, é possível identificar, até entre crianças, a formação de conhecimento mais genérico atuando sobre o processo de transferência. Ë provável que tais variações possam ser atribuídas a diferenças já apontadas entre as pesquisas, como domínio ou área de conhecimento, grau de 
similaridade entre os problemas usados e tipo de exposição ao análogo. Como veremos a seguir, o tipo de conhecimento anterior do participante também faz muita diferença, em virtude dos esquemas que são ativados na situação de pesquisa.

\section{Influência do Conhecimento: Conhecimento Anterior de Longo Prazo}

Algumas pesquisas que controlaram o conhecimento anterior dos participantes verificaram que quanto maior o conhecimento, maior acurácia da resposta na transferência, menor a influência de fatores como ausência de relação entre problemas, menor a influência de similaridade apenas superficial, menor consumo de tempo, melhor sensibilidade ao erro, menor persistência em estratégias inadequadas (Klhar \& Mc Coy Carver, 1988; Novick, 1988; Novick \& Holyoak, 1991). Foi verificada, também, maior uniformidade no processo de resolução de especialistas, enquanto iniciantes variaram mais neste aspecto. A comparação entre participantes avaliados como intermediários em experiência e iniciantes revelou maior monitoramento da própria atividade e simulação mental (Schraagen, 1993).

A influência de conhecimento anterior pode ocorrer até sem que o participante tenha consciência dela, por meio de ativação propagada (priming) de um conceito adquirido em outra situação (Schunn \& Dunbar, 1996). Porém, conhecimento anterior factual pode também prejudicar a transferência, quando o participante recorre às suas próprias teorias implícitas, às vezes inadequadas sobre um fenômeno (Catrambone, Jones, Jonides \& Seiffert, 1995), quando o enunciado do problema contém relações contra intuitivas entre os seus objetos (Simon \& Hayes, 1976), ou quando viola relações de superordenação ou subordinação, como por exemplo, distribuir vasos entre flores, secretárias entre computadores (Bassok, Chase \& Martin, 1998; Bassok, Wu \& Olseth, 1993), ou ainda, quando o participante mantém crenças limitadas acerca de recursos metacognitivos que podem auxiliá-lo na resolução de um problema (Antonietti, 1999). Entretanto, alguns outros recursos usados na instrução podem ajudar a diminuir a influência das teorias implícitas, como o estabelecimento de sub-objetivos ou a menção a uma analogia (Catrambone, 1994; Catrambone e cols., 1995) Outro aspecto de interesse é que quanto maior o conhecimento do participante sobre o domínio, menor a influência das violações ao que é usualmente encontrado na vida diária (Bassok e cols., 1993, Bassok e cols., 1998). Estes resultados indicam a necessidade de se estudar melhor a influência do conhecimento anterior sobre a transferência, porque limitam a necessidade da

Psicologia: Reflexão e Crítica, 2001, 14(3), pp. 521-536 similaridade, principalmente a superficial, para a percepção de analogia. Ao que tudo indica, os dados sobre similaridade podem estar contaminados pela pouca familiaridade dos participantes com o domínio. Estes problemas ficam mais evidenciados nos aspectos que examinaremos a seguir sobre as manipulações realizadas na etapa de aquisição que, de modo geral, atuam sobre a quantidade de informação adquirida pelo participante e seus efeitos sobre a transferência.

\section{Efeito da Informação Adicional Dada na Situação Experimental}

Como já apontado acima, um aspecto muito estudado nas pesquisas tem sido o efeito do fornecimento de informação adicional, seja na forma de dica ou pista acerca da similaridade entre os problemas, ou mesmo, sobre o princípio de resolução. As primeiras facilitam a recuperação do análogo no teste de transferência (Catrambone e cols., 1995; Chen, 1995; Gick \& Holyoak, 1980; Gick \& Holyoak, 1983), tornando a similaridade estrutural mais perceptível (Holyoak \& Koh, 1987). Um aspecto interessante é que a dica favorece a transferência em diferentes momentos: na aquisição, avisando-se o participante que irá usar a informação posteriormente (Stein e cols., 1986), no teste, e também em uma segunda oportunidade para transferir (Catrambone e cols., 1995; Chen, 1995; Gick \& Holyoak, 1980; Gick \& Holyoak, 1983;). A dica é mais efetiva se o contexto de aquisição for o mesmo (Spencer \& Weisberg, 1986). O tipo de pista, gráfica ou verbal não faz diferença, obtendo-se transferência com ambas (Gick, 1985; Chen, 1995) Quando há similaridade superficial entre os problemas, a dica pode ainda ser bastante genérica, referindo-se apenas ao que deve ser considerado (Stein e cols., 1986). Ela é efetiva, mesmo quando problemas distratores são intercalados entre os análogos, ou quando só um análogo é apresentado (Gick \& Holyoak, 1980). A dica ajuda na medida em que libera a memória do que não é importante para resolver o problema (Hesse \& Klecha, 1990). Nesta perspectiva, faz sentido a conclusão de que a similaridade favorece mais a transferência do que a regra porque, a primeira, ao preservar informação recorrente, sobrecarrega menos a memória (Wattenmaker, Mc Quaid, \& Schwarz, 1995).

Este último aspecto pode explicar os resultados obtidos com referência ao papel da regra ou outras informações gerais no processo de resolução. Verificouse que o fornecimento de informação relevante favorece mais a identificação da regra do que a descoberta da mesma porque sobrecarrega menos a memória (Kareev, Haberstald \& Shafir, 1993), quando é esclarecido como 
se deve raciocinar de modo consistente em problemas de lógica (Kroger e cols., 1997) ou, quando há prática extensiva (Wedman, Wedman \& Folger, 1996). Coerentemente com estes aspectos, verifica-se que o fornecimento de uma instrução geral (conselho), sobre como proceder em uma tarefa envolvendo analogia, e feedback sobre a correção da resposta durante a aquisição, também favorecem a transferência, pois o conselho ajuda na abstração do esquema e feedback na aprendizagem da tarefa (Phye, 1989; Phye, 1990; Phye \& Sanders, 1994). $\mathrm{Na}$ mesma linha, o fornecimento de uma legenda indicando a finalidade de uma seqüência de passos em um procedimento facilita a transferência porque esclarece o significado dos mesmos, criando um sub-objetivo (Catrambone, 1996). O que se pode concluir destes resultados é que informações, sejam gerais ou específicas, que ajudem a percepção do que é importante na situação, isto é, liberando a memória para a abstração do que é realmente similar, ajudam a transferência. Ё possível que a influência deste tipo de informação esteja sendo até super estimada, em função da artificialidade da situação de pesquisa, que como vimos, emprega problemas pouco relacionados à realidade do participante, não raro inseridos entre outras tarefas de pesquisa, ou mesmo problemas não relacionados entre si.

\section{Outros Facilitadores de Transferência}

A similaridade entre o contexto em que se dão a aquisição e teste (de mesma pesquisa versus pesquisas diferentes, ou pesquisa versus aula) também favorece a transferência (Catrambone \& Holyoak, 1989; Spencer \& Weisberg, 1986). Pensar em voz alta e justificar a seleção de uma dada ação na aquisição favorece a transferência, eliminando ações desnecessárias, acelerando o estabelecimento de procedimentos (Zook \& Di Vesta, 1989). É provável que o mesmo processo ocorra quando se dá ênfase à metacognição, via pensar em voz alta sobre próprios processos, pelo esforço em justificar as próprias ações (Berardi-Colleta, Buyer, Dominovski \& Rellinger, 1995). A ordem em que são apresentados os problemas também pode influir sobre a percepção de similaridade. Problemas mais complexos tornam mais fácil a transferência para problemas mais simples (Stein e cols., 1986). O que se pode concluir do exame destes últimos resultados é que as atividades que facilitam a percepção de similaridade entre os problemas e a abstração de esquema favorecem a transferência, na medida em que ativam somente a informação relevante. Examinaremos a seguir os resultados obtidos sobre a importância das diferentes etapas de processamento: representação, comparação ou mapeamento e adaptação sobre a transferência, que podem fornecer dados mais generalizáveis sobre este processo.

\section{Influência das Diversas Etapas de Processamento Sobre a Transferência \\ Representação}

A importância da representação no processo parece se limitar a esta etapa, pois treino fornecido para favorecer a aquisição de uma representação mais adequada à estrutura do problema não resultou em transferência de procedimento. Verificou-se ainda que a recuperação das formas mais adequadas de representar o problema é favorecida por similaridade superficial e por dica, não sendo afetada por similaridade somente estrutural (Novick \& Hemlo, 1994). Resultados esclarecedores para este tipo de limitação referem-se à suscetibilidade do processo de representação ao enunciado ou à questão proposta. Um enunciado inadequado dificulta o processo de resolução, ou porque sobrecarrega a memória com uma representação equivocada, que não corresponde ao estado desejado (Simon \& Hayes, 1976), ou porque a representação é interrompida assim que um procedimento é ativado, mesmo quando não é apropriado. Além disso, a interpretação da questão sofre influência de questões feitas em tarefas similares anteriores (Escarabajal \& Richard, 1986).

\section{Comparação}

O processo de comparação ou mapeamento é necessário, mas não suficiente para a transferência (Novick \& Holyoak , 1991). Isto porque ajuda no entendimento do problema, mas sofre influência da representação, do feedback, ou mesmo de problemas análogos anteriores, sendo, portanto, um processo dinâmico (Escarabajal \& Richard, 1986; Simon \& Hayes, 1976). Coerentemente com estes resultados e, também, com o que já foi analisado acima, é favorecido pelo estabelecimento de objetivos porque os mesmos favorecem a eliminação de informação não relevante, o que resulta em uma melhor codificação do problema (Spellman \& Holyoak, 1996), ou, então, uso de estratégias mais eficientes (Zook \& Di Vesta, 1989). Sua influência sobre a transferência parece variar segundo o tipo de tarefa, como, por exemplo, a abstração da regra que rege uma série. Nesta, o mapeamento dos predicados favoreceu a aplicação de uma relação analógica percebida em outra semelhante (Burns, 1996). Entre crianças, o processo de comparação feito no mapeamento, associado à exploração de espaço durante a aquisição favorece a transferência (Pierce e cols., 1993).

\section{Adaptação}

Já o processo de adaptação do procedimento não é automático e é melhor favorecido por pistas específicas, 
Tabela 1. Resumo dos Principais Resultados sobre Transferência Específica

\begin{tabular}{|c|c|c|}
\hline Variável & Resultado & Autores \\
\hline Manipulação nos Problemas & & \\
\hline Similaridade Total & $\begin{array}{l}\text { Facilitação da recuperação, adaptação e } \\
\text { aplicação }\end{array}$ & $\begin{array}{l}\text { Rickard e cols., 1994; Bard e cols., 1995; } \\
\text { Reed e cols., 1985; Patrick e Haines,1988 }\end{array}$ \\
\hline 1) Superficial & Recuperação da informação & $\begin{array}{l}\text { Holyoak e Koh, 1987; Ross, 1987, 1989; } \\
\text { Hesse e Klecha, } 1990\end{array}$ \\
\hline 2) Estrutural & $\begin{array}{l}\text { Adaptação e aplicação (raramente } \\
\text { espontâneas) }\end{array}$ & $\begin{array}{l}\text { Gick e Holyoak, 1980, Chen, 1995; } \\
\text { Gentner e Holyoak, 1997, Antonietti, } 199\end{array}$ \\
\hline
\end{tabular}

a) Problemas

b) Situação experimental

Relativas ao conhecimento

1) Anterior do sujeito:

a) Conhecimento de longo prazo Maioracurácia evelocidade sobre o problema

b) Conhecimento fatual

2) Adquirido na situação
Recuperação(risco de rigidez funcional ou Luchins, 1942; Duncker, 1945; Novick, 1988 transferência negativa) Recuperação Spencere Weisberg, 1986; Catrambone e Holyoak, 1985
Interferência negativa, neutralizada com maior conhecimento ou instruções

Informação simples, específica; declarativa

Conhecimento mais

abstrato(Esquemas)

\begin{tabular}{ll}
\hline Intervenções experimentais & Exigência de maior processamento: \\
1) Facilitação de esquema & resumos, comparação, instruções, etc.
\end{tabular}
Novick,1988; Klhar e Mc Coy Carver, 1988; Novick e Holyoak, 1991, Scraagen, 1993; Schunn e Dunbar,1996

Catrambone, 1994;Catrambone e cols., 1995; Simon e Haines, 1976; Bassok e cols.,1993; Bassok e cols., 1998; Antonietti, 1999

Reed e cols.,1974; Stein e cols., 1986; Anderson e Fichman, 1994

Spelman e Holyoak, 1996; Gick e Holyoak,1983; Catrambone e Holyoak,1989 Catrambone ,1994; Clement, 1994; Bernardo, 1994; Cooper e Sweeler, 1987; Kroger, Cheng e Holyoak, 1993; Hesse e Klecha, 1990; Novick e Holyoak, 1991

Clement,1994; Bernardo, 1994; Paas, 1992; Paas

eVan Merrinboer, 1994; Cooper e Sweeler, 1987; Gick e Holyoak, 1983; Pierce e cols., 1993; Pierce e cols., 1996; Novick e Holyoak, 1991

2) Informação adicional na situação experimental

a) Dica, regra Percepção de similaridade

b) Princípio de resolução

Adaptação quando explicita operações

Gick e Holyoak, 1980; Gick e Holyoak, 1983; Chen, 1995; Catrambone e cols., 1995; Holyoak e Koh, 1987; Stein e cols., 1987; Spencer e Weisberg, 1986; Gick, 1985; Hesse e Klecha, 1990; Kareev e cols., 1993; Kroger e cols., 1997; Wedman e cols., 1996; Phye, 1989; Phye, 1990; Phye e Sanders, 1994; Catrambone, 1996

Chen , 1995; Simon e Haines, 1976; Kotovsky e Simon, 1990; Hesse e Klecha, 1990

\begin{tabular}{ll}
\hline Outros: & Eliminam operações desnecessárias \\
Ênfase na metacognição & Acelera estabelecimento de procedimentos
\end{tabular}

Pensar em voz alta

Apresentação complexidade

Etapas de processamento

1) representação

suscetível à influência de outras variáveis

2) comparação

suscetível à influência de outras variáveis

3) adaptação

favorecida por pista específica
Zook e Di Vesta, 1989; Berardi-Colleta e cols., 1995; Stein e cols., 1986

Novick e Hemlo, 1994; Simon e Haines, 1976; Escarabajal e Richard, 1986

Novick e Holyoak, 1991; Simon e Haines, 1976; Escarabajal e Richard, 1986; Spelman e Holyoak, 1996; Zook e Di Vesta, 1989; Burns, 1996; Pierce e cols., 1993 Novick e Holyoak, 1991 
que explicitem as correspondências de operações a serem realizadas, do que por pistas conceituais, que apenas mostrem as semelhanças entre os problemas (Novick \& Holyoak, 1991). Verificamos, então, que nenhuma etapa do processamento garante por si só a transferência e que, além disso, não são automáticas, dependendo de um processamento deliberado por parte do participante. Estas etapas são, ainda, suscetíveis a outras influências como a formulação do enunciado, estabelecimento de objetivos e presença de informações como dicas e feedback.

\section{Transferência Geral}

Finalizando a análise de resultados das pesquisas cognitivistas, verificamos que poucas das mesmas, a maioria lidando com raciocínio lógico, ou atividades relacionadas como programação, obtiveram evidências de que a transferência pode ser geral e que a aquisição pode ser transferida para uma variedade mais ampla de situações. Tais resultados, como veremos a seguir, confirmam alguns pontos já apontados acima sobre transferência específica. Processos de aquisição que enfatizem a abstração de relações e que sejam adequados ao estilo de aprendizagem do participante podem favorecer esta transferência mais geral em Lógica (Stenning, Cox \& Oberlander, 1995). Em uma tarefa relacionada, de programação e avaliação de programas encontrou-se transferência considerável de uma tarefa para outra, principalmente no que diz respeito a conhecimento declarativo mais elaborado com a prática (Pennington e cols., 1995). Ainda no domínio da programação, foi verificado que crianças de oito a onze anos conseguem transferir habilidades gerais de solução de problemas e de programação. O sucesso da transferência foi atribuído ao tipo de medida usado para avaliá-la, uso da mesma habilidade em um domínio diferente do que foi adquirido (Klhar \& Mc Coy Carver, 1988). Com relação a este aspecto, transferência entre domínios diferentes, alguns resultados indicam que o processo sofre influência da natureza do mesmo. Equações aprendidas no contexto de problemas de Álgebra foram transferidas para problemas análogos de Física. Já as aprendidas neste último contexto não foram transferidas para problemas de Álgebra, sugerindo que as especificidades de alguns domínios são mais facilmente abstraídas do que outros (Bassok \& Holyoak, 1989).

Já em problemas de categorização, os resultados são menos conclusivos em relação à transferibilidade de procedimentos gerais, possivelmente em virtude da intervenção de outros fatores como natureza da própria tarefa ou estilo de resolução. Isto porque se verificou que, independente do tipo de instrução, algumas pessoas tendem a usar um tipo de estratégia mais geral, baseada em regra, enquanto outras usam uma mais específica, baseada em exemplos. Em tarefas de classificação, a estratégia analítica, baseada no uso de regra pode ser menos eficiente do que a analógica, baseada na busca de similaridades porque a atividade demanda coordenações de conhecimento anterior com o que pode ser extraído da situação. Nesta, a regra mostra-se menos eficiente porque não preserva informação recorrente ou correlacional. Entretanto, os dois tipos de estratégia podem interagir, dependendo das exigências da tarefa (Waltenmaker e cols., 1995). Mesmo com treino em um só tipo de estratégia, um mais analítico, focalizando partes do estímulo, ou outro mais sintético, codificando o todo formado pela série, verifica-se que o uso de uma ou outra depende do tipo de julgamento pedido ou da apresentação visual do estímulo. O processo parece envolver a interação entre fatores situacionais e de aplicação de conhecimento que, entretanto, podem ser investigados isoladamente, o que seria mais útil em problemas ricos em conhecimento e menos definidos (Withellesea, Brooks \& Westcott, 1994).

\section{Resultados das Pesquisas Realizadas com Outros Referenciais}

Como poderá ser observado a seguir, os resultados das pesquisas em outros referenciais confirmam algumas observações das pesquisas cognitivistas. Iniciando pelas pesquisas realizadas segundo o referencial behaviorista, verifica-se que a transferência é um fenômeno que goza de uma relativa independência das contingências ambientais. Estímulos treinados como membros de classes de equivalência não assumem o valor de reforçadores apenas com alguns treinos. Cerca de 40\% dos participantes não realizaram esta transferência, o que só foi obtido com outros treinos. Este resultado foi atribuído a algum tipo de controle exercido pelo contexto, que fez com que alguns estímulos treinados em um contexto não fossem transferidos para outro (Greenway e cols., 1996). Também no estudo de nomeação de figuras ocorreu dificuldade em se obter o desempenho esperado. A transferência só foi obtida quando se exigiu que o participante repetisse os nomes dos estímulos, e depois, apontasse o estímulo correspondente. Caso contrário, o participante dispersava sua atenção e só respondia próximo à apresentação (Glat e cols., 1994).

Já no estudo realizado segundo o referencial funcionalista, que visava a verificar como os conhecimentos que o participante adquire em uma tarefa são transferidos para a sua análoga mais complexa, foi observado que: a primeira tarefa propiciou a aquisição de algumas 
estratégias de ordenação, diferentes no número de inferências a serem realizadas; estas foram adaptadas para a tarefa mais complexa e ainda, a seleção das mesmas era feita não só em função da sua adequação, mas, também, da menor sobrecarga mental e melhor controle do procedimento que propiciava (Shao \& Nguyen- Xuan, 1993). É digna de nota a consistência que pode ser observada entre estes resultados e os objetivos que o referencial funcionalista se propõe alcançar, como, por exemplo, identificação de conhecimentos e estratégias dos participantes.

$\mathrm{Na}$ pesquisa sem referencial teórico verificou-se melhor transferência na condição poder manipular as peças e, também, na exposição decrescente de dificuldade, isto é, iniciar pelo problema mais complexo, que, porém, revelou-se menos econômica em termos de tempo de resolução (Tsai, 1987).

Concluindo, observa-se que os resultados das pesquisas realizadas em outros referenciais confirmam alguns resultados obtidos nas cognitivistas sobre a importância do contexto de aquisição e teste, da sobrecarga na memória e da ordem de apresentação dos problemas segundo a sua complexidade.

\section{Conclusão}

Como expusemos na introdução, o objetivo deste trabalho era examinar, por meio de uma análise das pesquisas sobre transferência de solução de problemas, as críticas feitas por Bruner (1990/1997) ao cognitivismo pelo desvio de sua proposta original, que resultou em uma abordagem fragmentada e tecnicista, perdendo de vista a investigação do significado. Em vista do que examinamos, consideramos que as críticas são procedentes na área de solução de problemas, principalmente nos aspectos destacados a seguir.

\section{Rigor Metodológico versus Teórico}

Observa-se um desequilíbrio entre rigor metodológico e teórico nas pesquisas cognitivistas, que realizam investigações experimentais muito bem controladas, provavelmente em virtude das críticas iniciais formuladas pelo behaviorismo. Entretanto, não se observa o mesmo rigor na parte conceitual, buscando-se uma definição mais precisa do fenômeno em investigação, no caso transferência de solução de problemas. Vimos que esta indefinição do conceito traz como conseqüência resultados muito fragmentados, quando não contraditórios sobre a própria ocorrência do fenômeno. Ainda a ressaltar, o que é verificado em situação de pesquisa contraria, muitas vezes, aquilo que sabemos que ocorre na vida cotidiana.

Psicologia: Reflexão e Crítica, 2001, 14(3), pp. 521-536
Em outras palavras, o ser humano transfere seu conhecimento para situações que deseja resolver, mas a pesquisa científica contribui pouco para desvendar como isso ocorre.

\section{Ênfase no Desempenho}

A grande maioria das pesquisas cognitivistas ao privilegiar o rigor metodológico preserva o modelo de investigação behaviorista, adotando uma posição empirista e tecnicista. Vimos que, na maioria das vezes, a transferência foi investigada em uma única ocasião, limitada na maioria das vezes ao desempenho de grandes grupos de participantes. Além disso, as condições experimentais altamente controladas, como a administração de instruções e problemas em folhetos é, além de artificial, de pouca validade ecológica, pouco generalizáveis para situações da vida real. Deixam ainda espaço para interpretações pessoais quanto aos reais objetivos da pesquisa, por serem pouco esclarecedoras, ou até enganadoras, usando problemas não relacionados entre si, ou mesmo, misturando tarefas de diferentes pesquisas. Já foi demonstrada que o significado atribuído pelos participantes ao que se objetiva investigar na pesquisa tem forte influência sobre os resultados (Schwartz, 1999). Ainda com relação a este aspecto do significado dado pelo participante à tarefa, vale lembrar o papel facilitador da dica ou pista sobre a transferência, evidenciado nos resultados. Ao que tudo indica, a tarefa experimental encontrava-se desprovida de significado para os participantes até o momento em que estas eram fornecidas. Entretanto, em nenhuma pesquisa foi verificada qualquer preocupação no sentido de verificar esta questão. Pelo contrário, observa-se maior preocupação com o delineamento experimental, com o tipo de similaridade entre materiais e procedimentos, que sugerem a crença subjacente no poder de determinação do estímulo sobre o comportamento. $\mathrm{O}$ uso de problemas que não exigem conhecimento anterior por mais da metade das pesquisas corrobora esta impressão, reforçada pela constatação do pouco interesse em investigar a influência de diferentes graus de conhecimento. Este último aspecto, quando investigado, limita-se na maioria das vezes a um controle de desempenho no Scholastic Aptitude Test (SAT), avaliação usada nos EUA para admissão nas universidades. Tal lacuna é lamentável, considerando os resultados sobre a menor necessidade de similaridade para a ocorrência de transferência entre os participantes com maior conhecimento do domínio.

\section{Utilização Preferencial de Estudantes Universitários}

Outro aspecto a destacar é o tipo de participante estudado na maioria adultos universitários. Muitas 
diferenças já foram detectadas entre processamento de informação infantil e adulto, tais como capacidade da memória de trabalho, capacidade de codificação, aproveitamento do feedback, habilidades metacognitivas entre outras (Carey, 1990; Sternberg, 2000). Além disto, como lembra Rosenthal (1970) ao citar a crítica feita por Mc Nemar, já na década de quarenta, sobre o recrutamento indiscriminado de participantes universitários nas pesquisas: "The existing science of human behavior is largely the science of the behavior of sophomores" (p.18). Segundo Rosenthal (1970), tal participação de um só tipo de participante, em geral, alunos de cursos introdutórios de Psicologia, que servem como voluntários neste tipo de estudo, não permite que se generalizem os resultados nem para a própria população de estudantes universitários. Isto porque a decisão em servir como voluntário em um experimento sofre influência de variáveis como: idade, verificando-se maior tendência entre os mais jovens a servir como voluntários; características de personalidade, como por exemplo, necessidade de aprovação; grau de conhecimento que o participante tem do experimentador, que quanto maior aumenta a probabilidade de participação.

\section{Resultados Aplicáveis à Educação}

Considerando os aspectos analisados acima, observase pouca preocupação com a generalização de resultados, obtidos em condições, já em si, pouco generalizáveis pela artificialidade em que foram obtidos. Quanto a estes últimos, reduzidos, em sua maioria, a dados quantitativos sobre o desempenho, verifica-se uma forte ênfase em tratamentos estatísticos, que pouco acrescentam, além da constatação que nada de muito novo foi desvendado, principalmente para a Educação: muito conhecimento, ou então, muita prática, muita variedade, muita informação através de instrução prévia, explicitação de similaridades, dicas, regras ou feedback.

\section{Referências}

Anderson, J. R. \& Fincham, J. M. (1994). Acquisition of procedural skills from examples. Journal of Experimental Psychology: Learning, Memory and Cognition, 20(6), 1322-1340.

Antonietti, A. (1991). Effects of partial analogies on solving an ill-defined problem. Psychological Reports, 68, 947-960.

Antonietti, A. (1999). Can students predict when imagery will allow them to discover the problem solution? European Journal of Cognitive Psychology, 11(3), 407-428.

Bard, C., Fleury, M., Gagnon, M., Michaud, D. Teasdale, N. \& Proteau, L. (1995). The transfer of perceptual and/or motor training to the performance of a coincidence-anticipation task. Journal of Experimental Child Psychology, 59, 32-48.
Bassok, M. \& Holyoak, K. J. (1989). Interdomain transfer between isomorphic topics in algebra and physics. Journal of Experimental Psychology: Learning, Memory and Cognition ,15(1), 153-166.

Bassok, M., Wu, L. L. \& Olseth, K. L. (1993). Judging a book by its cover: Interpretative effects of content on problem-solving transfer. Memory and Cognition 23(3), 354-367.

Bassok, M., Chase, V. M. \& Martin, S. A. (1998). Adding apples and oranges: Alignment of semantic and formal knowledge. Cognitive Psychology, 35, 99-104.

Berardi-Coletta, B., Buyer, L. S., Dominovski, R. L. \& Rellinger, E. R. (1995). Metacognition and problem solving: A process oriented approach. Journal of Experimental Psychology: Learning, Memory and Cognition, 21(1), 205-223.

Bernardo, A B. (1994). Problem-specific information and the development of problem-type schemata. Journal of Experimental Psychology: Learning, Memory and Cognition, 20(20), 379-395.

Ministério da Educação e do Desporto-Secretaria de Educação Fundamental. (1998). Parâmetros Curriculares Nacionais, 3 e 4 Ciclos do Ensino Fundamental. MEC, Brasilia, Brasil.

Burns, B. D. (1996) Meta-analogical transfer: Transfer between episodes of analogical reasoning. Journal of Experimental Psychology: Learning, Memory and Cognition 22(4), 1032-1048.

Bruner, J. (1997). Atos de significação (S. Costa Trad.). Porto Alegre: Artes Médicas. (Original publicado em 1990)

Carey, S. (1990). Cognitive development. Em D. P.Osherson \& E. Smith (Orgs.), An invitation to cognitive science - thinking (pp. 147-172). Cambridge: MIT.

Catrambone, R. (1994). Improving examples to improve transfer to novel problems. Memory and Cognition, 22(5), 606-615.

Catrambone, R. (1996). Generalizing solution procedures learned from examples. Journal of Experimental Psychology: Learning, Memory and Cognition, 22(4), 1020-1031.

Catrambone, R. \& Holyoak, K. (1989). Overcoming contextual limitations on problem solving transfer. Journal of Experimental Psychology: Learning, Memory and Cognition, 15(6), 1147-1156.

Catrambone, R., Jones, C. M., Jonides, J. \& Seiffert, C. (1995). Reasoning about curvilinear motion: Using principles or analogy. Memory \& Cognition, 23(3), 368-373.

Chen, Z. (1995). Analogical transfer: From schematic pictures to problem solving. Memory \& Cognition, 23(2), 225-269.

Clement, C. A. (1994). Effect of structural embedding on analogical transfer: Manifest versus latent analogs. American Journal of Psychology, 107(1), 1-38.

Cooper, G. \& Sweeler, J. (1987). Effects of schema acquisition and rule automation on mathematical problem solving transfer. Journal of Educational Psychology, 79(4), 347-362.

Coulet, J. C. (1999).Résolution de problèmes et theories du développement cognitif. Em M. Delau (Org.), Psychologie du développement (pp 103-146). Rosny Cedex: Bréal

Duncker, K. (1945). On problem solving. Psychological Monographs, 57(270), 1-113.

Fleury, M. T. L. \& Mattos, M. I. L. (1991). Sistemas educacionais comparados Estudos Avançados,12(5), 69-89.

Escarabajal, M. C. \& Richard, J. F. (1986). Le transfert analogique de procédures dans l'interpretation et la résolution d'un problème d'inclusion de classes chez des adultes. Archives de Psychologie, 54, 3964.

Gentner, D. \& Holyoak, K. J. (1997). Reasoning and learning by analogy Introduction. American Psychologist, 52(1), 32-34.

Gick, M. L. \& Holyoak, K. J. (1980). Analogical problem solving. Cognitive Psychology, 12, 306-335.

Gick, M. L. \& Holyoak, K. J. (1983). Schema induction and analogical transfer. Cognitive Psychology, 15, 1-38.

Gick, M. L. (1985). The effect of a diagram retrieval cue on spontaneous analogical transfer. Canadian Journal of Psychology, 39(3), 460-466. 
Glat, R., Gould, K., Stoddard, L. T. \& Sidman, M. (1994). A note on transfer of stimulus control in the delayed-cue procedure: Facilitation by an overt differential response. Journal of Applied Behavior Analysis, 27(4), 699-704.

Greenway, D. E., Dougher, M. J. \& Wulfert, E. (1996). Transfer of consequential functions via stimulus equivalence: Generalization to different testing contexts. The Psychological Record, 46, 131-143.

Hesse, F. W. \& Klecha, D. (1990). Use of analogies in problem solving. Computers in Human Behavior, 6, 115-129.

Holyoak, K. J. \& Koh, K. (1987) Surface and structural similarity in analogical transfer. Memory and Cognition, 15(4), 332-340.

Kareev, Y., Halberstadt, N. \& Shafir, D. (1993). Improving performance and increasing the use of non- positive testing in a rule-discovery task. The Quarterly Journal of Experimental Psychology, 46a, 729-742.

Klahr, D. \& McCoy Carver, S. (1988). Cognitive objectives in a Logo debugging curriculum: Instruction, learning, and transfer. Cognitive Psychology, 20, 362-404.

Kotovsky, K. \& Simon, H. A. (1990). What makes some problems really hard: Explorations in the problem space of difficulty. Cognitive Psychology, 22, 143-183.

Kroger, J. K., Cheng, P. W. \& Holyoak, K. J. (1993). Evoking the permission schema: The impact of explicit negation and a violation- checking context. The Quarterly Journal of Experimental Psychology, 46a(4), 615-635.

Luchins, A. S. (1942). Mechanization in problem solving: The effect of Einstellung. Psychological Monographs, 52, 248.

Maltzman, I. (1965). Thinking from a behavioristic point of view. Em R. C.Anderson \& D.P. Aususbel (Orgs.), Readings in the psychology of cognition (pp. 563-580). New York: Holt, Rinehart and Winston.

Mattos, M. I. L. (1993). Solução de problemas: Contribuições e impasses de três perspectivas teóricas: Gestalt, behaviorismo e cognitivismo. Estudos Avançados, Documentos, Série Ciência Cognitiva, 14.

Newell, A. \& Simon, H. (1972). Human problem solving. Englewood Cliffs, NJ: Prentice Hall.

Novick, L. R. (1988). Analogical transfer, problem similarity, and expertise. Journal of Experimental Psychology: Learning, Memory and Cognition, 14(3), 510-520.

Novick, L .R. \& Holyoak, K. J. (1991). Mathematical problem solving by analogy. Journal of Experimental Psychology: Learning, Memory and Cognition, 17(3), 398-415.

Novick, L. R. \& Hmelo, C. E. (1994) Transferring symbolic representations across nonisomorphic problems. Journal of Experimental Psychology: Learning, Memory and Cognition, 20(6), 1296-1321.

Paas, F. G. W. C. (1992). Training strategies for attaining transfer of problemsolving skill in statistics: A cognitive-load approach. Journal of Educational Psychology, 84(4), 429-434.

Paas, F. G. W. C. \& Van Merrinboer, J. J. G. (1994). Variability of worked examples and transfer of geometrical problem-solving skills: A cognitive-load approach. Journal of Educational Psychology, 86(1), 122 133.

Patrick, J. \& Haines, B. (1988). Training and transfer of faultfinding skill. Ergonomics, 31(2), 193-210.

Pennington, N., Nicolich, R. \& Rahm, J. (1995). Transfer of training between subskills: is knowledge use specific? Cognitive Psychology, 28, 175224.

Pierce, K. A., Duncan, M. K., Gholson, B., Ray, G. E. \& Khamhi, A. G. (1993). Cognitive load, schema acquisition, and procedural adaptation in nonisomorphic analogical transfer. Journal of Educational Psychology, $85(1), 66-74$.

Pierce, K. A. \& Gholson, B. (1994). Surface similarity and relational similarity in the development of analogical problem solving: Isomorphic and nonisomorphic transfer. Developmental Psychology, 30(5), 724-737.

Pierce, K.A, Crain, R. M., Gholson, B., Smither, D \& Rabinowitz, F. M. (1996). The sources of children's errors during nonisomorphic analogical transfer: Script theory and structure mapping theory. Journal of Experimental Child Psychology, 62, 102-130.
Phye, G. D. (1989). Schemata training and transfer of an intellectual skill. Journal of Educational Psychology, 81(3), 347-352.

Phye, G. D. (1990). Inductive problem solving: Schema inducement and memory-based transfer. Journal of Educational Psychology, 82(4), 826831.

Phye, G. D. \& Sanders, C. E. (1994). Advice and feedback: elements of practice for problem solving. Contemporary Educational Psychology, 19, 286-301.

Reed, S. K., Ernst, G. W. \& Banerii, B. (1974). The role of analogy in transfer between similar problem states. Cognitive Psychology, 6, 436-450.

Reed, S. K., Dempster, A. \& Ettinger, M. (1985). Usefulness of analogous solutions for solving algebra word problems. Journal of Experimental Psychology: Learning, Memory and Cognition, 11(1), 106-125.

Rickard, T.C., Healy, A. F. \& Bourne Jr., L. E. (1994). On the cognitive structure of basic arithmetic skills: Operation, order, and symbol transfer effects. Journal of Experimental Psychology: Learning, Memory and Cognition, 20(5), 1139-1153.

Robbins, R. W., Gosling, S. D. \& Craik, E. K. (1999) An empirical analysis of trends in psychology. American Psychologist, 54, 117-128.

Rosenthal, R. (1970). The volunteer subject. Em P. Badia, A. Haber \& R. P. Runyon (Orgs.), Research problems in psychology (pp. 18-41). Massachusetts: Addison Wesley.

Ross, B. H. (1987). This is like that: The use of earlier problems and the separation of similarity effects. Journal of Experimental Psychology: Learning, Memory and Cognition, 13(4), 629-639.

Ross, B. (1989). Distinguishing types of superficial similarities: Different effects on the acess and use of earlier problem. Journal of Experimental Psychology: Learning, Memory and Cognition, 15(3), 456-468.

Shao, J. \& Nguyen-Xuan, A. (1994). Inférence et résolution de problèmes: La seriation des poids avec une balance insensible. Archives de Psychologie, 62, 107-136.

Schraagen, J. M. (1993). How experts solve a novel problem in experimental design. Cognitive Science, 17, 285-309.

Schunn, C. D. \& Dunbar, K. (1996). Priming, analogy, and awareness in complex reasoning. Memory and Cognition, 24(3), 271-284.

Schwarz, N. (1999). Self-reports: How the questions shape the answers. American Psychologist, 54(2), 93-105.

Simon, H. \& Hayes, J. R. (1976). The understanding process: Problems isomorphs. Cognitive Psychology, 8, 191-216.

Skinner, B.F. (1966). Operant analysis of problem solving behavior. Em B. Kleinmutz (Org.), Problem solving research (pp. 225-257). New York: John Wiley \& Sons.

Skinner, B. F. (1989). The behavior of the listener. Em S. C. Hayes (Org.), Rule governed behavior cognition, contingencies and instructional control (pp. 8596). New York: Plenum Press.

Spellman, B. A. \& Holyoak, K. (1996). Pragmatics in analogical mapping. Cognitive Psychology, 31, 307-346.

Spencer, E. M. \& Weisberg, R. (1986). Context-dependent effects on analogical transfer. Memory \& Cognition, 14(5), 442-449.

Stein, B. S., Way, K. R., Benningfield, S. E. \& Hedgecough, C. A. (1986). Constraints on spontaneous transfer in problem- solving tasks. Memory \& Cognition, 14(5), 432-441.

Stenning, K., Cox, R. \& Oberlander, J. (1995). Contrasting the cognitive effects of graphical and sentential logic teaching: Reasoning, representation and individual differences. Language and Cognitive Processes, $10(3 / 4), 333-354$.

Sternberg, R. (2000). Psicologia cognitiva. Porto Alegre: Artes Médicas.

Tsai, L. S. (1987). Overt vs covert problem solving, transfer effects, and programming sequence I: Inverted triangles. Perceptual and Motor Skills, 65, 313-314.

Waltenmaker. W. D., McQuaid, H. L. \& Schwertz, S. J. (1995). Analogical versus rule - based classification. Memory \& Cognition, 23(4), 495-509.

Wedman, J., Wedman, J. \& Folger, T. (1996). Analysis of problem solving processes via think-aloud protocols. Journal of Research and Development in Education, 30(1), 51-62. 
Wertheimer, M. (1959). Productive thinking. New York: Plenum.

Whittlesea, B. W. A., Brooks, L. R. \& Westcott, C. (1994). After the learning is over: Factors controlling the selective application of general and particular knowledge. Journal of Experimental Psychology: Learning, Memory and Cognition, 20(2), 259-274.
Zook, K. B. \& Di Vesta, F. J. (1989). Effects of overt controlled verbalization and goal-specific search on acquisition of procedural knowledge in problem-solving. Journal of Educational Psychology, 81(2), 220-225.

Recebido: 26/12/2000

Revisado: $13 / 02 / 2001$

Aceite Final: 12/03/2001

Sobre a autora

Maria Isabel da Silva Leme é Doutora pelo Instituto de Psicologia da Universidade de São Paulo.

É Professora na Graduação do Curso de Psicologia da Universidade de São Paulo e no Programa de

Pós-Graduação em Psicologia Escolar e Desenvolvimento Humano da mesma instituição. 